\title{
Nonoperative Management of Penetrating Injuries to the Abdomen
}

\author{
Elizabeth Benjamin $^{1}$ • Demetrios Demetriades ${ }^{1}$
}

Published online: 8 April 2015

(C) Springer International Publishing AG 2015

\begin{abstract}
Contemporary management of penetrating abdominal trauma includes potential nonoperative management in a select group of patients. Patients with hemodynamic instability or peritonitis after penetrating abdominal trauma require immediate laparotomy. Stable, evaluable patients without peritonitis, however, may be selected for a protocol of serial abdominal and laboratory examinations. Cross-sectional imaging is a useful adjunct for trajectory mapping, especially after gunshot wounds. Although many patients may be successfully managed without operation, a subset of patients selected for nonoperative management will develop clinical signs of intra-abdominal injury and required delayed laparotomy. Protocols of selective nonoperative management after penetrating abdominal trauma have been shown to be safe. The desire to avoid the morbidity of nontherapeutic laparotomy, however, must continue to be weighed against the risk of missed intra-abdominal injury and the institutional feasibility of strict adherence to a protocol of serial examinations.
\end{abstract}

Keywords Selective nonoperative management .

Penetrating abdominal trauma

This article is part of the Topical Collection on Penetrating Injuries to Hollow Abdominal Viscera

Elizabeth Benjamin

Elizabeth.Benjamin@med.usc.edu

Demetrios Demetriades

Demetrios.Demetriades@med.usc.edu

1 LAC+USC Medical Center, University of Southern California, 2051 Marengo St, Inpatient Tower C5L-100, Los Angeles, CA 90033, USA

\section{Introduction}

The traditional teaching of mandatory surgical exploration for penetrating abdominal trauma was challenged in the 1960 s with the publication of the high nontherapeutic laparotomy rates associated with this practice [1]. The idea of selective nonoperative management of penetrating abdominal trauma initially gained support in patients with stab wounds; however, contemporary evaluation of abdominal gunshot injuries is also included in this algorithm.

A large percentage of patients with abdominal stab wounds have no clinically significant intra-abdominal injury. Demetriades et al. prospectively analyzed 467 patients with abdominal stab wounds and known peritoneal penetration and found that $27.6 \%$ of patients had no significant intraabdominal injury [2]. In a Western Trauma Association multicenter study of 359 patients with anterior abdominal stab wounds, only $36 \%$ of patients underwent a therapeutic laparotomy [3]. The authors concluded that, in the absence of hypotension, peritonitis, or evisceration, patients with anterior abdominal stab wounds could be observed for potential nonoperative management. These recommendations were validated in a follow-up study with no morbidity or mortality incurred by delayed operative intervention $[4 \bullet \bullet]$. Similar results have been reported after abdominal gunshot wounds. In a prospective study including 1856 patients, a policy of mandatory laparotomy after abdominal gunshot wound would have resulted in a nontherapeutic laparotomy rate of $47 \%$ [5]. Given these high rates of potential nontherapeutic intervention, the role of selective nonoperative management has been expanded to include both abdominal stab and gunshot wounds. 


\section{Selective Nonoperative Management}

The balance between nontherapeutic intervention and the morbidity of missed injury remains the cornerstone principle of selective nonoperative management. Similar to selective nonoperative management of solid organ injury after blunt trauma, not all patients selected for observation after penetrating abdominal trauma will avoid an operation. The critical component to safe selective nonoperative management is frequent and reliable physical examination. The potential morbidity of a nontherapeutic laparotomy is easily outweighed by the morbidity or potential mortality of a missed intraabdominal injury. Patients should be observed in a monitored setting with serial exams, ideally by the same, experienced team, in the absence of narcotics, antibiotics, or anesthesia. Hemodynamic and laboratory data, including white blood cell count, lactate, and serial hemoglobin, are monitored. Changes in the physical exam or hemodynamics should prompt critical re-evaluation for potential operative intervention. Although recent literature supports an observation period of 12-24 h, the majority of patients requiring laparotomy after penetrating abdominal trauma will manifest clinical symptoms within $4-6$ h $[6 \bullet \bullet, 7,8]$.

\section{Contraindications to Selective Nonoperative Management}

Hemodynamic instability and diffuse peritonitis following penetrating abdominal trauma remain absolute indications for exploratory laparotomy. In contrary to the localized tenderness that can be caused by the soft tissue damage of a superficial wound tract, diffuse peritonitis after penetrating abdominal trauma is associated with a high therapeutic laparotomy rate, even in the hemodynamically stable patient. In a review of 139 consecutive hemodynamically stable, peritonitic patients after penetrating abdominal trauma, $97 \%$ had intra-abdominal injury on laparotomy [9]. In addition, $39 \%$ required blood transfusion, and $25 \%$ developed intraoperative hypotension.

As accurate physical examination over time is imperative for safe nonoperative evaluation of a patient with penetrating abdominal trauma, altered level of consciousness, need for general anesthesia, or inability to perform reliable serial exams is a contraindication for selective nonoperative management.

\section{Safety of Selective Nonoperative Management}

Although injuries will rarely be missed with a policy of uniform laparotomy after penetrating trauma, the potential morbidity of nontherapeutic laparotomy is as high as $20 \%$ [ 10 , 11], with the later complications of hernias and bowel obstructions likely underreported. Nontherapeutic laparotomy has also been associated with increased hospital length of stay and cost $[5,12]$. With the advent of selective nonoperative management for penetrating abdominal trauma, the reservation lies in the patient that converts from the nonoperative to operative arm and whether or not the delay in operation contributes to patient morbidity and mortality. Delayed intervention, however, when following a protocol of serial abdominal and laboratory examinations, has not been shown to result in significant adverse outcome due to the prompt identification of clinical deterioration $[4 \bullet \bullet, 10,13-15,16 \bullet \bullet$.

\section{Imaging}

Radiologic imaging, including cross-sectional imaging, is a central component to the workup of a patient with penetrating abdominal trauma. Although the decision for operative intervention is largely based on clinical findings, imaging is an integral part of mapping the missile trajectory after penetrating injury.

\section{X-ray}

Plain X-rays are critical in the evaluation of missile trajectory. External examination will reveal entry and/or exit wounds; however, in the setting of gunshot injury, knowledge of the presence and location of retained fragments is essential to the understanding of bullet trajectory and for the triage of potential injuries. Paired flank wounds, for example, may represent a single, extraperitoneal tract, or two transabdominal or even transthoracic tracts with retained missiles.

\section{Focused Abdominal Sonography for Trauma}

Although focused abdominal sonography for trauma (FAST) has become a critical component of the evaluation of the blunt trauma patient, its utility after penetrating trauma remains limited. In patients with potential cardiac injury based on missile trajectory, the FAST is a highly sensitive tool for identifying pericardial fluid with the exception of patients that decompress the hemopericardium into the left chest through an open pericardial injury $[17,18]$. For abdominal wounds, FAST is specific for detecting intra-abdominal fluid but has low sensitivity for predicting operatively significant injury [19-21]. A positive FAST may be reflective of a solid organ injury that could be managed without operation while a negative FAST may miss a hollow viscus injury. For this reason, FAST exam should not be used as the sole indication for operative intervention. In patients with hypotension and multi-cavitary penetrating trauma, ultrasound, including the thoracic windows, may be a useful adjunct for operative decision-making.

\section{Computed Tomography}

Although the utility of computed tomography (CT) scan after abdominal stab wounds remains uncertain, the associated air 
bubble tract of a gunshot injury allows for trajectory mapping and potential identification of associated injury [22, 23••, 24]. Overall, CT scan is thought to have a sensitivity and specificity of 90.5 and $96 \%$ in detecting operatively significant injuries after abdominal gunshot wounds. Further, the presence of an extra-abdominal or solid organ trajectory can precipitate discharge or change in disposition $[15,25,26]$. Solid organ injury alone is not an indication for operative intervention, and the rates of successful nonoperative management in these patients are high, especially with the increasing utilization of angioembolization techniques $[15,27]$.

Although radiologic adjuncts can be integral to the evaluation of patients with penetrating abdominal trauma, the decision to proceed with operative intervention in the stable patient remains driven by physical examination. In a subset of patients with anterior abdominal stab wounds that underwent operative intervention based on test results, there was a $45 \%$ nontherapeutic laparotomy rate $[4 \bullet \bullet]$. In a prospective analysis of stable patients with abdominal stab wounds, excluding patients that qualified for immediate laparotomy or discharge, 177 patients were evaluated by both serial physical examination and CT scan. All patients requiring therapeutic laparotomy had physical exam findings precipitating operative intervention leading to a sensitivity and specificity of the physical exam of 100 and $98.7 \%$, respectively [23••].

\section{Selective Nonoperative Management Algorithm}

Patients with penetrating abdominal trauma may be divided into two distinct groups. First are the patients that present with hemodynamic instability, peritonitis, or are unevaluable (Fig. 1). This group of patients should be taken immediately to the operating room for exploration. Stable patients are then evaluated for possible imaging. Although, CT scan after abdominal stab wounds is not universally accepted, crosssectional imaging is frequently utilized at our center. If it is not obtained, and there is no concern for solid organ injury, serial abdominal exams are performed as part of the selective nonoperative management algorithm. CT imaging after abdominal gunshot wounds treated without operative intervention is clearly indicated and universally performed. CT imaging will then yield four possible groups. First, patients that clearly have intra-abdominal pathology on imaging require immediate operative intervention. Second, patients with fully extra-abdominal trajectory may be considered for potential discharge. Third, patients with isolated solid organ injury can be triaged to observation, potential angioembolization, or operative intervention depending on the radiologic findings and clinical progression. Finally, patients with concern for intra-abdominal trajectory but unclear injury can be observed. This is the primary group of patients that constitutes the selective nonoperative management cohort, relying on serial physical examinations and laboratory data to guide management. Patients that have operatively significant intra-abdominal injury will become clinically apparent within $24 \mathrm{~h}$. If patients decline clinically or develop diffuse peritonitis, they should be immediately re-evaluated for transition to operative intervention.

\section{Left Thoracoabdominal Penetrating Trauma}

Penetrating trauma to the left thoracoabdominal region from the nipple and scapular tip to the costal margin has potential to result in diaphragmatic injury in as many as $17-40 \%$ of patients [28-30]. Although patients with left diaphragmatic injury are often asymptomatic on presentation, there is a potential for wound expansion and delayed diaphragmatic hernia. For this reason, patients that have successfully undergone
Fig. 1 Algorithm for selective nonoperative management

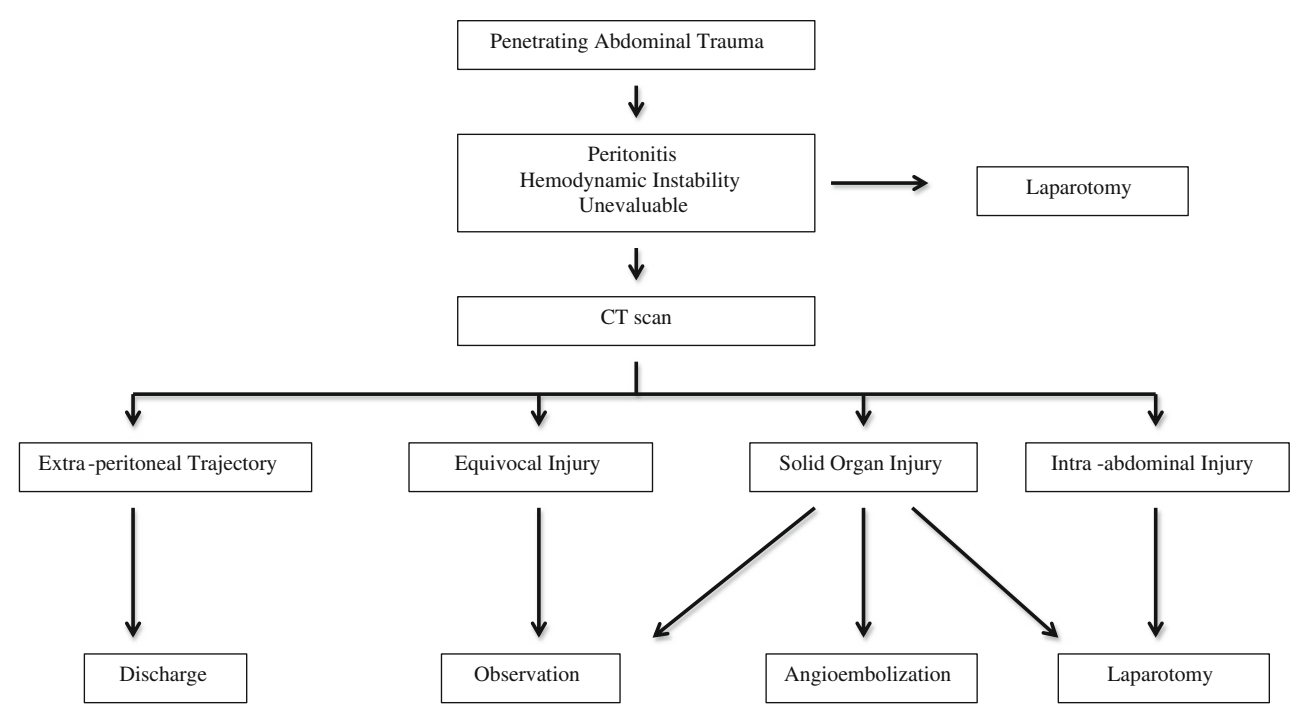


nonoperative management of left thoracoabdominal stab wounds should be offered a diagnostic laparoscopy or thoracoscopy to evaluate the diaphragm prior to discharge. Injuries to the diaphragm can be repaired using minimally invasive techniques.

\section{Summary}

Selective nonoperative management can be safely applied to patients with penetrating abdominal trauma. The desire to avoid nontherapeutic laparotomy, however, must be tempered with a clear understanding of the clinical signs and symptoms that signify failure of nonoperative management and the need for conversion to operative intervention. Serial abdominal exams remain the cornerstone of selective nonoperative management; however, radiologic and laboratory studies can be useful adjuncts in the decision-making process.

\section{Compliance with Ethics Guidelines}

Conflict of Interest Elizabeth Benjamin and Demetrios Demetriades declare that they have no conflicts of interest.

Human and Animal Rights and Informed Consent This article does not contain any studies with human or animal subjects performed by any of the authors.

\section{References}

Papers of particular interest, published recently, have been highlighted as:

•- Of major importance

1. Shaftan GW. Indications for operation in abdominal trauma. Am J Surg. 1960;99:657-64.

2. Demetriades D, Rabinowitz B. Indications for operation in abdominal stab wounds. A prospective study of 651 patients. Ann Surg. 1987;205(2):129-32.

3. Biffl WL, Kaups KL, Cothren CC, Brasel KJ, Dicker RA, Bullard $\mathrm{MK}$, et al. Management of patients with anterior abdominal stab wounds: a Western Trauma Association multicenter trial. J Trauma. 2009;66(5):1294-301.

4.• Biffl WL, Kaups KL, Pham TN, Rowell SE, Jurkovich GJ, Burlew CC, et al. Validating the Western Trauma Association algorithm for managing patients with anterior abdominal stab wounds: a Western Trauma Association multicenter trial. J Trauma. 2011;71(6):1494502 . Validation study for the safety and effectiveness of a protocol of serial clinical assessments for patients with penetrating abdominal stab wounds.

5. Velmahos GC, Demetriades D, Toutouzas KG, Sarkisyan G, Chan LS, Ishak R, et al. Selective nonoperative management in 1,856 patients with abdominal gunshot wounds: should routine laparotomy still be the standard of care? Ann Surg. 2001;234(3):395-402. discussion -3 .

6.• Inaba K, Branco BC, Moe D, Barmparas G, Okoye O, Lam L, et al. Prospective evaluation of selective nonoperative management of torso gunshot wounds: when is it safe to discharge? J Trauma Acute Care Surg. 2012;72(4):884-91. Prospective analysis that demonstrates early clinical findings in patients that fail selective nonoperative management for abdominal gunshot wounds. Sets a recommendation of a minimum 24 hour observation period.

7. Alzamel HA, Cohn SM. When is it safe to discharge asymptomatic patients with abdominal stab wounds? J Trauma. 2005;58(3):5235 .

8. MacLeod J, Freiberger D, Lewis F, Feliciano D. What is the optimal observation time for a penetrating wound to the flank? Am Surg. 2007;73(1):25-31.

9. Brown CV, Velmahos GC, Neville AL, Rhee P, Salim A, Sangthong B, et al. Hemodynamically "stable" patients with peritonitis after penetrating abdominal trauma: identifying those who are bleeding. Arch Surg. 2005;140(8):767-72.

10. Leppäniemi A, Salo J, Haapiainen R. Complications of negative laparotomy for truncal stab wounds. J Trauma. 1995;38(1):54-8.

11. Renz BM, Feliciano DV. Unnecessary laparotomies for trauma: a prospective study of morbidity. J Trauma. 1995;38(3):350-6.

12. Renz BM, Feliciano DV. The length of hospital stay after an unnecessary laparotomy for trauma: a prospective study. J Trauma. 1996;40(2):187-90.

13. Tsikitis V, Biffl WL, Majercik S, Harrington DT, Cioffi WG. Selective clinical management of anterior abdominal stab wounds. Am J Surg. 2004;188(6):807-12.

14. Leppäniemi AK, Haapiainen RK. Selective nonoperative management of abdominal stab wounds: prospective, randomized study. World J Surg. 1996;20(8):1101-5. discussion 5-6.

15. Demetriades D, Hadjizacharia $\mathrm{P}$, Constantinou $\mathrm{C}$, Brown $\mathrm{C}$, Inaba $\mathrm{K}$, Rhee $\mathrm{P}$, et al. Selective nonoperative management of penetrating abdominal solid organ injuries. Ann Surg. 2006;244(4):620-8.

16.• Peev MP, Chang Y, King DR, Yeh DD, Kaafarani H, Fagenholz PJ, et al. Delayed laparotomy after selective non-operative management of penetrating abdominal injuries. World J Surg. 2015;39(2): $380-6$. Systematic review of patients over an eight year period that underwent selective nonoperative management of penetrating abdominal trauma. The authors highlight the importance of a structured protocol with serial examinations and demonstrate the safetyof this protocol with early recognition of change in clinical status and need for operative intervention.

17. Ball CG, Williams BH, Wyrzykowski AD, Nicholas JM, Rozycki GS, Feliciano DV. A caveat to the performance of pericardial ultrasound in patients with penetrating cardiac wounds. J Trauma. 2009;67(5):1123-4.

18. Rozycki GS, Feliciano DV, Ochsner MG, Knudson MM, Hoyt DB, Davis F, et al. The role of ultrasound in patients with possible penetrating cardiac wounds: a prospective multicenter study. J Trauma. 1999;46(4):543-51. discussion 51-2.

19. Soffer D, McKenney MG, Cohn S, Garcia-Roca R, Namias N, Schulman C, et al. A prospective evaluation of ultrasonography for the diagnosis of penetrating torso injury. J Trauma. 2004;56(5):953-7. discussion 7-9.

20. Udobi KF, Rodriguez A, Chiu WC, Scalea TM. Role of ultrasonography in penetrating abdominal trauma: a prospective clinical study. J Trauma. 2001;50(3):475-9.

21. Boulanger BR, Kearney PA, Tsuei B, Ochoa JB. The routine use of sonography in penetrating torso injury is beneficial. J Trauma. 2001;51(2):320-5.

22. DuBose J, Inaba K, Teixeira PG, Pepe A, Dunham MB, McKenney $M$. Selective non-operative management of solid organ injury following abdominal gunshot wounds. Injury. 2007;38(9):1084-90.

23.• Inaba K, Okoye OT, Rosenheck R, Melo N, Branco BC, Talving P, et al. Prospective evaluation of the role of computed tomography in the assessment of abdominal stab wounds. JAMA Surg. 2013;148(9):810-6. Prospective analysis validating the high 
sensitivity and specificity of physcial examination to determine patients that require operative intervention after abdominal stab wounds.

24. Salim A, Sangthong B, Martin M, Brown C, Plurad D, Inaba K, et al. Use of computed tomography in anterior abdominal stab wounds: results of a prospective study. Arch Surg. 2006;141(8): 745-50. discussion 50-2.

25. Velmahos GC, Constantinou C, Tillou A, Brown CV, Salim A, Demetriades D. Abdominal computed tomographic scan for patients with gunshot wounds to the abdomen selected for nonoperative management. J Trauma. 2005;59(5):1155-60. discussion 60-1.

26. Navsaria PH, Nicol AJ, Krige JE, Edu S. Selective nonoperative management of liver gunshot injuries. Ann Surg. 2009;249(4):653-6.
27. Berg RJ, Inaba K, Okoye O, Pasley J, Teixeira PG, Esparza M, et al. The contemporary management of penetrating splenic injury. Injury. 2014;45(9):1394-400.

28. Murray JA, Demetriades D, Asensio JA, Cornwell EE, Velmahos GC, Belzberg H, et al. Occult injuries to the diaphragm: prospective evaluation of laparoscopy in penetrating injuries to the left lower chest. J Am Coll Surg. 1998;187(6):626-30.

29. Murray JA, Demetriades D, Cornwell EE, Asensio JA, Velmahos G, Belzberg H, et al. Penetrating left thoracoabdominal trauma: the incidence and clinical presentation of diaphragm injuries. J Trauma. 1997;43(4):624-6.

30. Leppäniemi A, Haapiainen R. Occult diaphragmatic injuries caused by stab wounds. J Trauma. 2003;55(4):646-50. 